\title{
Possibility of a metallic phase in granular superconducting films
}

\author{
Tai Kai $\mathrm{Ng}^{1}$ and Derek K. K. Lee ${ }^{2}$ \\ ${ }^{1}$ Department of Physics, Hong Kong University of Science and Technology, Clear Water Bay, Hong Kong, People's Republic of China \\ ${ }^{2}$ Blackett Laboratory, Imperial College, Prince Consort Road, London SW7 2BW, United Kingdom \\ (Received 10 July 2000; revised manuscript received 22 November 2000; published 19 March 2001)
}

\begin{abstract}
We investigate the possibility of finding a zero-temperature metallic phase in granular superconducting films, modeled as a dissipative Bose system. We extend the conventional treatment of these systems to a formalism that can distinguish between insulator, metal, and superfluid phases. We do not find a metallic state at zero temperature. At finite temperatures, we find that the system exhibits a crossover between superfluid and metallic behavior. This has implications for the analysis of experimental results that makes use of a scaling hypothesis involving only a single parameter. We also investigate the effect of vortex dissipation in these systems.
\end{abstract}

DOI: 10.1103/PhysRevB.63.144509

PACS number(s): 74.76. $-\mathrm{w}, 74.80 . \mathrm{Bj}, 74.25 . \mathrm{Dw}$

\section{INTRODUCTION}

Recently, there has been renewed interests in the problem of superconductor-insulator (SI) transition in low- $T_{c}$ thin films. These systems undergo transition from superconductor to insulator as a function of disorder, film thickness, or applied magnetic field ${ }^{1}$. Theories ${ }^{2,3}$ describing this kind of superconductor-insulator transition describe a second-order quantum phase transition where a zero-temperature metallic phase exists as a critical point between the superfluid and insulating phases. However, recent experiments ${ }^{4,5}$ found that the metallic phase may be more than a point in the phase diagram in certain systems. Instead, the zero-temperature conductivity appears to be finite and nonzero in a finite region in the phase diagram. It remains controversial whether these systems remain metallic down to zero temperature. It has been observed ${ }^{6}$ that some of these systems become superconducting at very low temperatures.

In this paper, we reexamine our theoretical understanding of the SI transition. ${ }^{7,8} \mathrm{We}$ will use a variational treatment which, in principle, may describe superfluid, metallic, and insulator phases at zero temperature. We will discuss dissipation arising from normal resistance or vortex motion. We find that, although there may be a direct SI transition at zero temperature, finite-temperature crossover phenomena may give rise to apparently metallic behavior in experiments. We also see that the two dissipation mechanisms affect the lowtemperature behavior on different sides of the SI critical point.

The destruction of the superconducting state at zero temperature is a result of strong Coulomb interactions. Consider a lattice model for Cooper pairs. Strong Coulomb repulsion leads to a Mott insulating state where there is an integral number of Cooper pairs at each site. However, if the system is coupled to a normal fluid, any excess charge on a site (arising the motion of Cooper pairs from site to site) can be screened to a certain extent by the normal component. This is effective when the normal fluid has low resistance $R_{n}$, because it can respond rapidly to charge fluctuations. Since the coupling to the normal fluid requires exchange of energy, the normal fluid can be regarded as a dissipative environment for the Cooper pairs. The strength of this dissipative coupling (or dynamic screening) is inversely proportional to $R_{n}$.

We will also consider dissipation originating from the motion of the normal cores in vortices. ${ }^{9,10}$ In this case, a similar picture applies when we study the system in a dual representation where vortices are the elementary bosonic objects.

In principle, dissipation may lead to nonsuperfluid but mobile Cooper pairs (or vortices) at zero temperature. ${ }^{11}$ To investigate this issue, we require a formulation that can differentiate between the superfluid, metallic, and insulating states. We will see below that we can do so by considering separately local phase fluctuations that, over a time scale of $\hbar / k_{\mathrm{B}} T$, are small compared to $2 \pi$ and those which are larger than $2 \pi$. Previous work has investigated either a superfluidto-nonsuperfluid transition $^{12}$ or an insulator-to-conductor transition. ${ }^{13} \mathrm{We}$ want to see if these transitions are separate so that all three phases exist. Otherwise, they are different descriptions of the same critical point, in which case the Bose metal does not exist in the model at zero temperature. After establishing the ground state, we will also discuss the finite-temperature behavior of these systems.

\section{DISSIPATIVE BOSE MODEL}

For simplicity, we will consider first dissipation for the Cooper pairs. Vortex dissipation will be discussed later. We will review the conventional discussion of this problem and we extend previous treatments by a more careful consideration of large phase fluctuations.

As our starting point, we use a model of superconducting grains on a square array. We assume that well-defined Cooper pairs exist in each grain so that we can treat them as charge- $2 e$ bosons. An imaginary-time action that describes the coupling between grains is ${ }^{14}$ :

$$
S_{\text {boson }}=\int_{0}^{\beta} d \tau\left[\frac{1}{2 K_{b}} \sum_{i}\left(\dot{\theta}_{i}^{b}\right)^{2}-J_{b} \sum_{i v} \cos \Delta_{\nu} \theta_{i}^{b}\right]
$$

where $\theta_{i}^{b}$ is the local superconducting phase of grain $i$, and $\Delta_{\nu} \theta_{i}^{b}=\theta_{i+\nu}^{b}-\theta_{i}^{b} . J_{b}$ is the Josephson coupling energy between nearest-neighbor grains. $K_{b}=2 e^{2} / C$ is the charging energy of a grain with self-capacitance $C$. (We have set $\hbar$ 


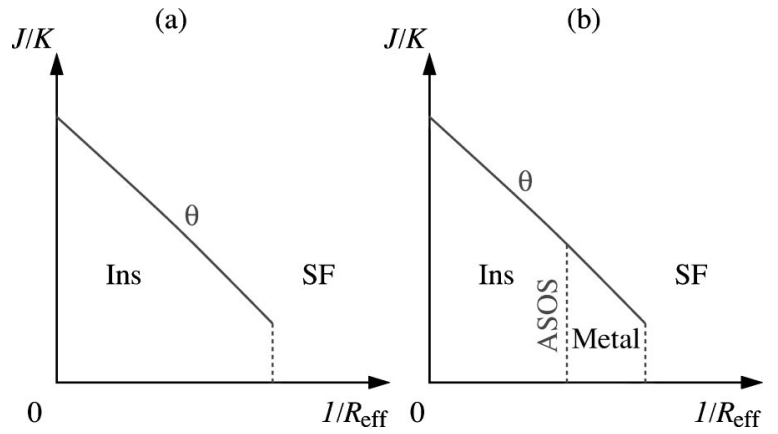

FIG. 1. Possible scenarios at zero temperature. Ins, insulator; SF, superfluid. Solid and dashed lines denote first- and second-order transitions. ASOS line, transition for winding numbers in the absolute solid-on-solid model. $\theta$ line denotes transition for small fluctuations $\theta$, as given in Ref. 12.

$=k_{\mathrm{B}}=1$, and $\beta=1 / T$ is the inverse temperature.) For large $J_{b} / K_{b}$, we expect a superconductor with long-range phase coherence. When $J_{b} / K_{b}$ is small, however, the on-site repulsion dominates and we have a Mott insulator. (Our calculations below will focus on this limit.) The system becomes incompressible. (See vertical axis on Fig. 1.) The phase $\theta^{b}$ of the local superconducting order parameter should fluctuate strongly at each site due to the number-phase uncertainty relation.

We will now investigate the effect of dissipation on this bosonic Mott transition. We include dissipation phenomenologically. We assume that an action of the Caldeira-Leggett kind $^{15,16}$ is necessary so that the charge currents $\left(\sim \Delta_{\nu} \theta^{b}\right)$ will have ohmic decay in the classical limit,

$$
\begin{aligned}
S_{\mathrm{diss}}= & \frac{Q^{2}}{2} \sum_{i \nu} \int_{0}^{\beta} d \tau \int_{0}^{\beta} d \tau^{\prime} \alpha\left(\tau-\tau^{\prime}\right) \\
& \times \sin ^{2}\left[\frac{\Delta_{\nu} \theta_{i}^{b}(\tau)-\Delta_{\nu} \theta_{i}^{b}\left(\tau^{\prime}\right)}{2 Q}\right],
\end{aligned}
$$

where $\alpha(\tau)=\left(h / 4 e^{2} R_{n}\right)[T / \sin (\pi T \tau)]^{2}$ and $Q=2$ reflecting the fact that the Cooper pair has charge $2 e$ while the dissipation is due to charge-e electrons.

We will be interested in the destruction of superfluidity due to enhanced phase fluctuations. As already mentioned, we have to be careful about the compactness of the phase variables $\theta_{i}^{b}$. The imaginary-time evolution of the phase can be separated into a periodic part $\theta_{i}$ and a nonperiodic part, ${ }^{3}$

$$
\theta_{i}^{b}(\tau)=\frac{2 \pi n_{i} \tau}{\beta}+\theta_{i}(\tau)+\theta_{0 i},
$$

where $\theta_{i}(\beta)=\theta_{i}(0)$ and $\theta_{0 i}$ is a constant. The boson action can be written as

$$
\begin{aligned}
S_{\mathrm{boson}}= & \frac{2 \pi^{2}}{\beta K_{b}} \sum_{i} n_{i}^{2}+\frac{1}{2 K_{b}} \sum_{i} \int_{0}^{\beta} \dot{\theta}_{i}^{2} d \tau \\
& -J_{b} \sum_{i \nu} \int_{0}^{\beta} \cos \left(\Delta_{\nu} \theta_{i}(\tau)+\frac{2 \pi \tau}{\beta} \Delta_{\nu} n_{i}\right) d \tau,
\end{aligned}
$$

To further simplify our calculation, we shall assume strong dissipation and keep only the $\Delta_{\nu} \theta_{i}$ terms in $S_{\text {diss }}$ to second order. At low temperatures, we obtain, ${ }^{13}$

$$
\begin{aligned}
S_{\mathrm{diss}} \rightarrow & \frac{Q \pi}{4 R_{n}} \sum_{i, \nu}\left|\Delta_{\nu} n_{i}\right|+\frac{1}{8} \sum_{i \nu} \int_{0}^{\beta} d \tau \int_{0}^{\beta} d \tau^{\prime} \alpha\left(\tau-\tau^{\prime}\right) \\
& \times \cos \left[\frac{2 \pi\left(\tau-\tau^{\prime}\right)}{Q \beta} \Delta_{\nu} n_{i}\right]\left[\Delta_{\nu} \theta_{i}(\tau)-\Delta_{\nu} \theta_{i}\left(\tau^{\prime}\right)\right]^{2} .
\end{aligned}
$$

We can now discuss possible scenarios for the zerotemperature phase diagram of the system. First of all, let us concentrate on the part of the action that involves only the "winding numbers" $n_{i}$. We can ignore the charging term in Eq. (4) proportional to $n_{i}^{2}$ because it vanishes as $T \rightarrow 0$. The winding numbers are controlled by the first term in Eq. (5). This is, in fact, the "absolute solid-on-solid" (ASOS) model that has a "roughening" $\operatorname{transition}^{17}$ (of the KosterlitzThouless type) at $R_{n}=R_{c}^{\mathrm{ASOS}} \simeq 0.6\left(h / Q e^{2}\right)$. For large $R_{n}$, the phase at each site fluctuates wildly with little correlation between different sites. This is what is expected (from number-phase uncertainty) in an insulator where the local particle number does not fluctuate. For small $R_{n}$, the system becomes "smooth" in the sense that large excursions in the phase are suppressed. The system is now compressible and the charges are mobile. This model has been used to describe an insulator-conductor transition in normal tunneling junction networks when $R_{n}$ is small enough. ${ }^{13}$

We have seen that the Mott insulator breaks down and charges are mobile at small $R_{n}$. What about superfluidity for these mobile charges? This requires long-range phase coherence in the system. In other words, in addition to a "smooth" $n$ field, the fluctuations of $\theta$ at different sites must also be coherent. Therefore, in principle, we may have a superfluid or metallic state for these mobile charges, depending on whether the phase stiffness for $\theta$ fluctuations is finite or not.

If we ignore the coupling of the $\theta$ field to the winding numbers $n_{i}$, then we expect a superfluid at small $R_{n}$ at $T$ $=0 .\left(R_{n}<h / 2 e^{2}\right.$ in two dimensions. $\left.{ }^{12}\right)$ A primary purpose of this paper is to investigate whether the onset of a finite phase stiffness for $\theta$ coincides with the appearance of the smooth phase in the SOS model for the winding number [i.e., a direct superfluid-insulator transition, as shown in Fig. 1(a).] Another scenario is that a metal phase exists for intermediate values of $R_{n}$ where the ASOS model is smooth before longrange phase coherence sets in at an even lower value of $R_{n}$ [Fig. 1(b)].

The actions (4) and (5) form the basis of our calculations. The model cannot be solved exactly even without the dissipative term. We shall pursue a variational approach since we are only interested at the qualitative behavior of the system-in particular, whether a zero-temperature metallic phase exists under appropriate conditions. We consider the following trial action, $S_{0}=S_{\theta}+S_{\mathrm{MSOS}}$, 


$$
\begin{aligned}
S_{0}^{\theta}= & \sum_{i} \int_{0}^{\beta} d \tau\left[\frac{1}{2 K_{b}} \dot{\theta}_{i}^{2}+\frac{J_{\mathrm{eff}}}{2} \sum_{\nu}\left(\Delta_{\nu} \theta_{i}\right)^{2}\right] \\
& +\frac{1}{8} \sum_{i \nu} \int_{0}^{\beta} d \tau \int_{0}^{\beta} d \tau^{\prime} \alpha_{\mathrm{eff}}\left(\tau-\tau^{\prime}\right) \\
& \times\left[\Delta_{\nu} \theta_{i}(\tau)-\Delta_{\nu} \theta_{i}\left(\tau^{\prime}\right)\right]^{2}, \\
S_{0}^{\mathrm{MSOS}}= & \frac{2 \pi^{2}}{\beta K_{b}} \sum_{i} n_{i}^{2}+\sum_{i \nu}\left[\frac{Q \pi}{4 R_{\mathrm{eff}}}\left|\Delta_{\nu} n_{i}\right|-\beta J_{\mathrm{MS}} \delta_{\Delta_{\nu} n_{i}}\right]
\end{aligned}
$$

where $\alpha_{\text {eff }}(\tau) / \alpha(\tau)=R_{n} / R_{\text {eff }} . J_{\text {eff }}, J_{\mathrm{MS}}$, and $R_{\text {eff }}$ are parameters to be determined variationally. Note that the solid-onsolid part of the model has been modified by the presence of the $J_{\mathrm{MS}}$ term. Similar to the other terms in the ASOS model, it also suppresses the spatial fluctuations in the winding number. We therefore expect this modified solid-on-solid (MSOS) model to be similar to the ASOS model with a shifted critical point $R_{c}^{\mathrm{MSOS}}$.

The possibilities of superconductor, insulator, and metal phases at zero temperature are all included in $S_{0}$. A finite value for the phase stiffness $J_{\text {eff }}$ indicates that we have a superconductor (marked 'SF' in Fig. 1). If $J_{\text {eff }}=J_{\mathrm{MS}}=0$, then the system is nonsuperfluid. To determine whether it is an insulator or a metal, we examine the large phase fluctuations, i.e., the MSOS model for the winding numbers. The system is an insulator if $R_{\text {eff }}>R_{c}^{\mathrm{MSOS}}$ so that the winding numbers are in the rough phase. If $R_{\mathrm{eff}}<R_{c}^{\mathrm{MSOS}}$, the winding-number fluctuations are smooth and we have a metallic state. [See Fig. 1(b). Note that the ASOS and MSOS models are the same if $J_{\text {eff }}=J_{\mathrm{MS}}=0$.]

The variational parameters are determined by minimizing the free energy per unit volume given approximately by $F$ $=F_{0}+\left\langle S_{\text {boson }}+S_{\text {diss }}-S_{0}\right\rangle_{0} / \beta L^{2}$, where $F_{0}$ is the free energy calculated using $S_{0}$ and $\langle\cdots\rangle_{0}$ denotes averages taken with respect to $S_{0}$. We obtain the mean-field equations,

$$
\begin{gathered}
R_{\mathrm{eff}}=R_{n}, \\
J_{\mathrm{MS}}=J_{b} e^{-\left\langle|\Delta \theta|^{2}\right\rangle / 2,} \\
J_{\mathrm{eff}}=J_{\mathrm{MS}} P_{\mathrm{SOS}}(0),
\end{gathered}
$$

where

$$
\left\langle|\Delta \theta|^{2}\right\rangle=\frac{1}{2 \beta L^{2}} \sum_{q, i \omega_{n}} \gamma(\vec{q}) G_{0 \theta}\left(\vec{q}, i \omega_{n}\right),
$$

with $G_{0 \theta}^{-1}\left(\vec{q}, i \omega_{n}\right)=\omega_{n}^{2} / K_{b}+\gamma(\vec{q})\left(J_{\text {eff }}+\left|\omega_{n}\right| / 4 R_{\text {eff }}\right) . \quad \gamma(\vec{q})$ $=4\left[\sin ^{2}\left(q_{x} / 2\right)+\sin ^{2}\left(q_{y} / 2\right)\right]$ is the lattice-dispersion relation. $P_{\mathrm{SOS}}(m)=\left\langle\delta\left(\left|\Delta_{\nu} n_{i}\right|-m\right)\right\rangle_{\mathrm{MSOS}}$ is the probability that the nearest-neighbor integer difference $\left|\Delta_{\nu} n_{i}\right|=m$ in the MSOS model. Note that we can also regard our trial action as a "Hartree" decoupling of the fields $\theta_{i}$ and $n_{i}$.

For the small phase fluctuations, the critical point for the onset of a finite $J_{\text {eff }}$ is given by Chakravarty et al. ${ }^{12} R_{c}^{\theta}$ $=h / Q e^{2} . J_{\text {eff }}$ vanishes when $R_{\text {eff }}>R_{c}^{\theta}$ and, for $R_{\text {eff }}<R_{c}^{\theta}$, it

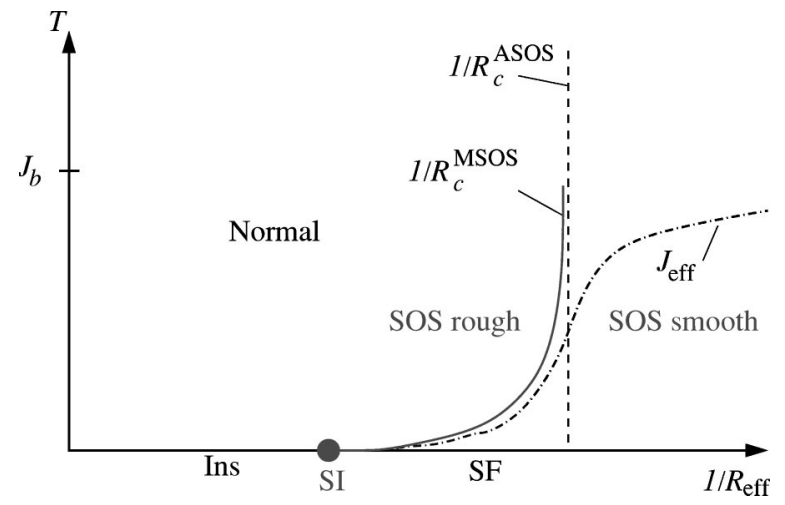

FIG. 2. Phase diagram. A superfluid-insulator critical point (SI) separates the superfluid (SF) and insulating (Ins) phases at $T=0$. The system is normal at finite temperatures. The solid line indicates the roughening transition of the winding numbers in the solid-onsolid (MSOS) sector of the model. The vertical dashed line denotes the corresponding line for the ASOS model $\left(J_{\mathrm{MS}}=J_{\text {eff }}=0\right)$. The dash-dotted line denotes the dependence of $J_{\text {eff }}$ on $R_{\text {eff }}$. Our treatment is valid on the right of the solid line (see Sec. IV).

approaches its bare value $J_{b}$ away from the transition. Near the transition, $J_{\text {eff }}$ becomes exponentially small,

$$
\frac{J_{\text {eff }}}{J_{b}} \sim\left(\frac{J_{b} R}{K_{b}}\right)^{R_{c}^{\theta} /\left(R_{c}^{\theta}-R_{\text {eff }}\right)} \quad\left(R_{\text {eff }} \longrightarrow R_{c}^{\theta}\right)
$$

This is indicated in Fig. 2.

To determine the ground-state properties of the system, we also need to examine the SOS sector of the model. We see that the $J_{\mathrm{MS}}$ term dominates the MSOS model at low temperatures, and so the SOS sector is smooth whenever $J_{\text {eff }}$ is finite. When $J_{\text {eff }}$ vanishes, we find that $R_{n}$ is already above the critical value for the SOS critical point (i.e., $R_{c}^{\theta}$ $>R_{c}^{\mathrm{ASOS}}$ ). Therefore, the winding-number sector is always rough when $J_{\text {eff }}=0$ so that the system is an insulator. This means that, at the level of this mean-field calculation, we cannot have a metallic phase at zero temperature. We see that the system has only one quantum-critical point as we change $R_{n}$ (marked "SI" in Fig. 2): $R_{c}=R_{c}^{\theta}=R_{c}^{\mathrm{MSOS}}$. This corresponds to the scenario in Fig. 1(a).

\section{FINITE TEMPERATURE}

We will now discuss the system at finite temperature. We confine our discussion to the low-temperature regime where $T \leqslant J_{b} \ll K_{b}$. For simplicity, we will also focus on the case where the magnetic penetration depth is finite (small compared to the system size) so that, at any finite temperature, vortices have short-ranged interactions and are unbound and the system is not superconducting. We will see that the winding-number fluctuations have important consequences for the behavior of the system because the MSOS model governing these fluctuations has an apparent finitetemperature phase transition. We will argue that these effects show up as crossover behavior as the system is cooled to zero temperature.

First of all, we note that the mean-field treatment ${ }^{12}$ of the 
Gaussian fluctuations in $S_{0}^{\theta}$ gives a finite-temperature phase transition for the loss of phase stiffness at $T_{c} \simeq J_{\mathrm{MS}}(T=0)$ (see dashed line in Fig. 2). For a neutral superfluid, this will be a Kosterlitz-Thouless transition. We are studying a charged superconductor with a finite penetration depth. There will be no sharp transition but only a crossover, marking the energy scale below which the correlation length $\xi_{\theta}$ for the small phase fluctuations becomes large compared to the lattice spacing. Using the large- $N$ expansion of an $\mathrm{O}(N)$ model,${ }^{18}$ we expect this correlation length to diverge at zero temperature: $\xi_{\theta}(T) \sim\left(K / J_{b}\right)^{1 / 2}\left(J_{b} / T\right) \exp \left(2 \pi J_{\text {eff }} / T\right)$ for $T$ $\ll J_{\text {eff }}$ when $R_{\text {eff }}<R_{c}^{\theta}$ (to the right of the "SI" point in Fig. 2).

However, before we can understand overall phase coherence in the system, we also have to study how the windingnumber fluctuations behave at finite temperatures. This is controlled by $S_{0}^{\mathrm{MSOS}}$. We will see that there is also an apparent phase transition in these quantities.

Consider first the case when $J_{\mathrm{MS}}=0$, in other words, when there is no stiffness in the small-phase fluctuations $\theta$, i.e., to the left of the "SI" point in Fig. 2. The winding number is then governed by an ASOS model (the second term in $S_{0}^{\mathrm{MSOS}}$ ) at low temperatures. This has a roughening transition whose position does not change with temperature. This is indicated by the vertical dashed line in Fig. 2. We see that $J_{\mathrm{MS}}=0$ falls in the rough phase of the ASOS model. Thus, there is no long-range coherence in both the large- and small-phase fluctuations when $J_{\mathrm{MS}}=0$. As discussed in the previous section, this is an insulating state at $T=0$.

The above discussion is a good description of the winding numbers as long as the temperature is high: $\beta J_{\mathrm{MS}} \ll 1$, i.e., above and to the left of the dash-dotted line in Fig. 2. However, at temperatures below $J_{\text {eff }}$, the $\beta J_{\mathrm{MS}}$ term becomes important in $S_{0}^{\mathrm{MSOS}}$. (Note that $J_{\mathrm{MS}}$ and $J_{\text {eff }}$ differ by a numerical factor of order unity that is noncritical across the roughening transition. We will use them interchangeably when we discuss energy scales in this section.) The windingnumber fluctuations are even further suppressed in this regime compared to the ASOS model. [This term alone gives us the Potts model that has a transition when $\beta J_{\mathrm{MS}} \sim O(1)$.] Therefore, we expect $R_{c}^{\mathrm{MSOS}}$ to increase from the ASOS critical value at low temperatures (solid line in Fig. 2.) So, we obtain a smooth phase for the MSOS model for $T<J_{\text {eff }}$. This $J_{\mathrm{MS}}$-dominated regime shrinks as we go towards $T=0$. At $T=0, \quad J_{\text {eff }}$ vanishes when $R_{\text {eff }}=R_{c}^{\theta}$, and so we expect $R_{c}^{\mathrm{MSOS}}(T=0)$ to coincide with $R_{c}^{\theta}$, as discussed in the previous section (Fig. 2). In other words, for a resistance in the region $0.6 Q<Q^{2} e^{2} R_{n} / h<2$, the MSOS sector will cross a roughening transition for the winding numbers as we decrease the temperature.

Again, we expect that this transition is an artifact of the variational treatment in the case of our superconductor with a finite penetration depth. We believe that it will manifest itself as a crossover phenomenon in the system. This is because, while there appears to be two correlation lengths in this formulation $\left(\xi_{\theta}\right.$ and $\xi_{\text {Sos }}$ for the small and large fluctuations, respectively), there is only one true phase-correlation length, $\xi$. The winding-number correlation length $\xi_{\text {SOS }}$ di- verges at finite temperature when we cross the roughening transition $\left[R_{\text {eff }}=R_{c}^{\mathrm{MSOS}}(T)\right]$. The true correlation length is given by,

$$
\xi\left(R_{\mathrm{eff}}, T\right)=\min \left[\xi_{\mathrm{SOS}}\left(R_{\mathrm{eff}}, T\right), \xi_{\theta}\left(J_{\mathrm{eff}}, T\right)\right] .
$$

So, the SOS model does not give rise to a divergence in observable quantities, since any divergence in the SOS model will be cut off when $\xi_{\text {Sos }}$ becomes comparable to $\xi_{\theta}(T)$ that is finite at all nonzero temperatures. Therefore, we conclude that the SOS roughening transition is smoothed out and we see a broad crossover behavior as we traverse the apparent SOS transition line in Fig. 2.

If there is no actual roughening transition, why should we be concerned with the winding-number fluctuations? This is because the above discussion alerts us that physical quantities would not have a simple dependence on the temperature $T$ and the proximity of the resistance to the critical value, $R_{n}-R_{c}$. In the conventional Gaussian theory, we can use a scaling form that simply depends on $\xi_{\theta}$. However, our discussion shows that the system also depends on $\xi_{\text {SOS }}$ that has a different functional dependence on $R_{\text {eff }}$ and $T$.

This tells us that single-parameter scaling might fail near the SOS transition line. In particular, we expect that the scaling behavior of physical quantities would be different on the two sides of the SOS transition line. Of course, we should recover single-parameter scaling for data well below the SOS transition line. However, this is not experimentally accessible near the zero-temperature critical point since $J_{\text {eff }}$ becomes very small in this regime [see Eq. (10)]. This also makes it hard to pinpoint the position of the superfluidinsulator transition experimentally.

We believe that these considerations are a significant source of difficulties for the scaling analysis of experimental data, and may be responsible for the observation of an apparent metallic phase in some experiments. ${ }^{4,5}$

To be cautious, we should stress that this result depends on the observation that $R_{c}^{\theta}>R_{c}^{\mathrm{ASOS}}$ [see discussion below Eq. (10)] so that it is sensitive to our estimates of $R_{c}^{\theta}$ and $R_{\text {eff }}$. For instance, we note that $R_{n}$ is unrenormalized in our variational equations (8). A more careful treatment of the dissipative term might renormalize this quantity and therefore shift the relative positions of the critical points of the $\theta$ and SOS sectors. We will assume that these estimates are correct in the next section.

\section{BEYOND GAUSSIAN FLUCTUATIONS}

The above analysis is based on a treatment that treats the coupling between the small- and large-phase fluctuations ( $\theta$ and $n$ ) in a Hartree-like manner. In this section, we will check that this is reasonable by considering higher-order fluctuations. We will see that the crossover effect mentioned above shows up as the breakdown of our Hartree-like decoupling of the small- and large-phase fluctuations.

To consider higher-order fluctuations, let us examine the free energy density $f$. This can be written as 


$$
\begin{aligned}
f-f_{0} & =-\ln \left\langle\exp \left[-\left(S-S_{0}\right)\right]\right\rangle_{0} / \beta L^{2} \\
& \simeq\left[\left\langle S-S_{0}\right\rangle_{0}-\left\langle\left(S-S_{0}\right)^{2}\right\rangle_{c 0} / 2\right] / \beta L^{2}+\cdots,
\end{aligned}
$$

where averages are taken with respect to the trial action $S_{0}$ and $\langle A B\rangle_{c}=\langle A B\rangle-\langle A\rangle\langle B\rangle$ denotes the connected part of the correlation function. Minimizing the first term in this expansion gives the variational treatment in the previous section. To consider the validity of this approach, we should check that higher-order terms do not diverge. These correspond to fluctuations beyond the Hartree-like treatment in the previous section. We restrict our attention to the first correction.

We can separate the Josephson and dissipative parts of $S-S_{0}$ as $\delta S^{\mathrm{J}}+\delta S^{\mathrm{D}}$ where

$$
\begin{gathered}
\delta S^{\mathrm{J}}=\sum_{i \nu} \int_{0}^{\beta} d \tau\left[-J_{b} \cos \left(\Delta_{\nu} \theta_{i}+\frac{2 \pi \tau}{\beta} \Delta_{\nu} n_{i}\right)\right. \\
-\frac{J_{\mathrm{eff}}}{2}\left(\Delta_{\nu} \theta_{i}\right)^{2}+J_{\mathrm{MS}} \delta_{\left.\Delta_{\nu} n_{i}\right]} \\
\delta S^{\mathrm{D}}=\sum_{i \nu} \int_{0}^{\beta} d \tau \int_{0}^{\beta} d \tau^{\prime} \alpha\left(\tau-\tau^{\prime}\right) \\
\times\left[\cos \left(\frac{2 \pi\left(\tau-\tau^{\prime}\right)}{Q \beta} \Delta_{\nu} n_{i}\right)-1\right]\left[\Delta_{\nu} \theta_{i}(\tau)-\Delta_{\nu} \theta_{i}\left(\tau^{\prime}\right)\right]^{2}
\end{gathered}
$$

As we approach the SI transition $\left(J_{\text {eff }} \rightarrow 0, R_{\text {eff }} \rightarrow R_{c}\right)$ at zero temperature, we find that the singular part of $\left\langle\left(\delta S^{\mathrm{J}}\right)^{2}\right\rangle / \beta L^{2}$ comes from fluctuations in $\theta$, scaling as $J_{\text {eff }}^{2 R_{\text {eff }} / R_{c}-1}$. We see that $\left\langle\left(\delta S^{\mathrm{J}}\right)^{2}\right\rangle$ does not diverge even at the critical point. In the nonsuperfluid phase $\left(J_{\text {eff }}=0\right)$, these fluctuations are proportional to $T$ as $T \rightarrow 0$.

The contributions to $\left\langle\delta S^{\mathrm{J}} \delta S^{\mathrm{D}}\right\rangle / \beta L^{2}$ are also finite, scaling as $T$ when $T \rightarrow 0$ at finite $J_{\text {eff }}$, and scaling as $J_{\text {eff }}$ when $J_{\text {eff }}$ $\rightarrow 0$ at finite $T$.

We find that the most singular term comes from $\left\langle\left(\delta S^{\mathrm{D}}\right)^{2}\right\rangle$. Let $A_{\tau, \tau^{\prime}}=\Sigma_{i \nu}\left[\Delta_{\nu} \theta_{i}(\tau)-\Delta_{\nu} \theta_{i}\left(\tau^{\prime}\right)\right]^{2}$ and $B_{\tau, \tau^{\prime}}=\Sigma_{i \nu} \alpha(\tau$ $\left.-\tau^{\prime}\right)\left[\cos \left\{2 \pi\left(\tau-\tau^{\prime}\right) \Delta_{\nu} n_{i} / Q \beta\right\}-1\right] / 2$. Then, the contribution from

$$
\begin{aligned}
I & =\frac{1}{\beta L^{2}} \int\left\langle A_{\tau_{1}, \tau_{1}^{\prime}}\right\rangle\left\langle A_{\tau_{2}, \tau_{2}^{\prime}}\right\rangle\left\langle B_{\tau_{1}, \tau_{1}^{\prime}} B_{\tau_{2}, \tau_{2}^{\prime}}\right\rangle_{c} d \tau_{1} d \tau_{1}^{\prime} d \tau_{2} d \tau_{2}^{\prime} \\
& \sim \frac{1}{\beta} \sum_{\omega \omega^{\prime}} \frac{\sum_{\mathbf{r} \mu \nu}\left\langle g_{\mu}(\mathbf{r}, \omega) g_{\nu}\left(\mathbf{0}, \omega^{\prime}\right)\right\rangle_{c}}{\left(4 J_{\mathrm{eff}} R_{\mathrm{eff}}+|\omega|\right)\left(4 J_{\mathrm{eff}} R_{\mathrm{eff}}+\left|\omega^{\prime}\right|\right)} \\
= & \sum_{\substack{\omega>2 \pi T\left|\Delta_{\nu} n_{\mathbf{r}}\right| \\
\omega^{\prime}>2 \pi T\left|\Delta_{\mu} n_{0}\right|}} \frac{T^{3} \sum_{\mathbf{r} \mu \nu}\left\langle\left|\Delta_{\nu} n_{i}\right|\left|\Delta_{\mu} n_{0}\right|\right\rangle_{c}}{\left(4 J_{\mathrm{eff}} R_{\mathrm{eff}}+|\omega|\right)\left(4 J_{\mathrm{eff}} R_{\mathrm{eff}}+\left|\omega^{\prime}\right|\right)}
\end{aligned}
$$

where $g_{\nu}\left(\mathbf{r}=\mathbf{r}_{i}, \omega\right)=\min \left(\omega, 2 \pi Q^{-1} T\left|\Delta_{\nu} n_{i}\right|\right)$. The numerator is a connected correlation function for the MSOS model.
We expect it to have exponential decay with correlation length $\xi_{\text {SOS }}$ in the smooth phase (and power-law decay in the rough phase).

In the smooth phase of the MSOS model where $J_{\text {eff }}$ is also finite, we see that $I \sim T \xi_{\text {SOS }}^{2} \ln \left(K_{b} / J_{\text {eff }}\right)$ as $T \rightarrow 0$. On the other hand, we expect the quantity $\sum_{i j \mu \nu}\left\langle\left|\Delta_{\nu} n_{i}\right|\left|\Delta_{\mu} n_{j}\right|\right\rangle_{c}$ to have the same critical behavior as the energy fluctuations-it diverges as we cross the line of SOS critical points. As discussed in the previous section, this will not be a true divergence, but only a crossover. Nevertheless, this means that this contribution from $\left\langle\left(\delta S^{\mathrm{D}}\right)^{2}\right\rangle$ will be large if we cross the SOS transition line as we raise the temperature in the superfluid phase.

This marks the breakdown of our treatment of the phase fluctuations in this model (in the region to the left of the dashed line in Fig. 2). However, since no divergences occur if we work at zero temperature, the conclusion of a direct superfluid-insulator transition appears robust (subject to the remarks at the end of the previous section about the accuracy of our estimates of the relative values for the critical points for the two sectors of the model).

\section{VORTEX DISSIPATION}

We will now discuss dissipation by vortex motion. Microscopically, this is due to the motion of the normal vortex core. We will, however, follow a phenomenological approach here.

For this purpose, it is convenient to study the system in a vortex representation. Fluctuations can be described by vortex loops in Euclidean space time. In particular, the superfluid state for the Cooper pairs corresponds to a vortex insulator $^{2}$ where there is a gap to the addition of a vortexthe Meissner effect. Conversely, the duality transformation shows that the Meissner phase of the vortices correspond to an insulating state for Cooper pairs (i.e., there is a gap to density excitations.)

To obtain the vortex representation, a duality transformation can be applied to the action (1) to obtain the dual action $S_{\text {dual }}=S_{A}+S_{\mathrm{v}}$ for vortices, where ${ }^{19}$

$$
\begin{aligned}
& S_{A}=\sum_{i} \int_{0}^{\beta} d \tau\left[\frac{1}{2 J_{b}}\left|(\nabla \times \vec{A})^{s}\right|_{i}^{2}+K_{b}\left|(\nabla \times \vec{A})^{\tau}\right|_{i}^{2}\right], \\
& S_{\mathrm{v}}=\int_{0}^{\beta} d \tau\left[\frac{1}{2 K_{v}} \sum_{i}\left(A_{i}^{\tau}-\dot{\theta}_{i}^{v}\right)^{2}-J_{v} \sum_{i \nu} \cos D_{\nu} \theta_{i}^{v}\right]
\end{aligned}
$$

where $D_{\nu} \theta_{i}=\Delta_{\nu} \theta_{i}-A_{i}^{\nu}$ is a covariant derivative. The internal gauge field $A$ is defined so that $\nabla \times \vec{A}$ is the boson 3 current. Its action $S_{A}$ describes the phonons in the (original) boson superfluid. (The superscripts $s$ and $\tau$ denote the spatial and temporal components respectively.) The action $S_{\mathrm{v}}$ describes vortices in the system: $\theta_{i}^{v}$ is the phase of the vortex wave function on site $i$ of the dual lattice. We have introduced the terms $K_{v} \sim J_{b}$ and $J_{v} \sim 2 e \sqrt{J_{b} / c}$ to characterize the core energy and the hopping integral of the vortices, 
respectively. ${ }^{19}$ The coupling of the vortex phase to the gauge field expresses the fact that vortices are advected by the current of the original bosons.

The qualitative behavior of the system should not depend on details of the vortex interaction as long as it is short ranged. We therefore choose the lattice spacing $d$ for the dual model to be of the order of the penetration depth (of the original Cooper pairs), and include only on-site repulsion for vortices. For simplicity, we choose a square lattice. (To obtain a finite penetration depth explicitly from this duality transformation, we should include a coupling of the original bosons to the real external gauge field and add the conventional magnetic field energy to the Hamiltonian. A shortranged interaction between vortex densities is obtained on integrating out this gauge field.)

As with the boson model discussed above, we expect the system to have a superfluid-insulator transition as we increase $K_{v} / J_{v}$. To include dissipation phenomenologically, we again assume that an action of the Caldeira-Leggett kind $^{15,16}$ so that the vortex currents $\left(\sim D_{\nu} \theta^{v}\right)$ will decay with a decay rate proportional to the current,

$$
\begin{aligned}
S_{\mathrm{diss}, \mathrm{v}}= & \frac{1}{2} \sum_{i \nu} \int_{0}^{\beta} d \tau \int_{0}^{\beta} d \tau^{\prime} \alpha\left(\tau-\tau^{\prime}\right) \\
& \times \sin ^{2}\left[\frac{D_{\nu} \theta_{i}^{v}(\tau)-D_{\nu} \theta_{i}^{v}\left(\tau^{\prime}\right)}{2}\right],
\end{aligned}
$$

where $\alpha(\tau)=\left(h / 4 e^{2} R_{v}\right)[T / \sin (\pi T \tau)]^{2}$ with $4 e^{2} R_{v} / h \sim(1$ $-t) / t$ and $t \sim e^{-\eta d^{2} / \hbar}$ is the tunneling resistance of vortices from one grain to another. ${ }^{10}$ Note that we have set $Q=1$ in this case because we do not have a microscopic reason for the dissipative mechanism to involve objects with a charge that is different from the bosons. The vortex viscous drag coefficient $\eta$ is given by $\Phi_{o} H_{c 2} / R_{n} c^{2}$ where $R_{n}$ is the normal-state resistance of the superconductor, $H_{c 2}$ is the upper critical field, and $\Phi_{0}=h c / 2 e$ is the flux quantum. The details of the relationship between $R_{v}$ and $R_{n}$ are not important here. Suffice to note that they are inversely related to each other and comparable when both are of the order of $h / e^{2}$. The coupling to the internal gauge field is required by gauge invariance.

We see that this model is similar to the one discussed in the previous sections, except that the bosons are now coupled to an internal gauge field. We can again separate the imaginary-time evolution of the phase into a periodic part, $\theta_{i}(\tau)$, and a nonperiodic part, $2 \pi n_{i} \tau / \beta$, (3) to obtain

$$
\begin{aligned}
S_{\mathrm{dual}}= & S_{A}+\frac{2 \pi^{2}}{\beta K_{v}} \sum_{i} n_{i}^{2}+\frac{1}{2 K_{v}} \sum_{i} \int_{0}^{\beta} \dot{\theta}_{i}^{2} d \tau \\
& -J_{v} \sum_{i \nu} \int_{0}^{\beta} \cos \left(D_{\nu} \theta_{i}(\tau)+\frac{2 \pi \tau}{\beta} \Delta_{\nu} n_{i}\right) d \tau .
\end{aligned}
$$

Repeating the treatment in the previous sections (with $Q$ $=1$ ), we have the trial action,

$$
\begin{aligned}
S_{0}= & \sum_{i} \int_{0}^{\beta} d \tau\left[\frac{1}{2 K_{v}} \dot{\theta}_{i}^{2}+\frac{J_{\mathrm{eff}}}{2} \sum_{\nu}\left(D_{\nu} \theta_{i}\right)^{2}\right] \\
& +\frac{1}{8} \sum_{i \nu} \int_{0}^{\beta} d \tau \int_{0}^{\beta} d \tau^{\prime} \alpha_{\mathrm{eff}}\left(\tau-\tau^{\prime}\right) \\
& \times\left[D_{\nu} \theta_{i}(\tau)-D_{\nu} \theta_{i}\left(\tau^{\prime}\right)\right]^{2}+\frac{2 \pi^{2}}{\beta K_{v}} \sum_{i} n_{i}^{2} \\
& +\sum_{i \nu}\left[\frac{\pi}{4 R_{\mathrm{eff}}}\left|\Delta_{\nu} n_{i}\right|-\beta J_{\mathrm{MS}} \delta_{\Delta_{\nu} n_{i}}\right] .
\end{aligned}
$$

The variational parameters are now given by $R_{\text {eff }}=R_{v}, J_{\text {eff }}$ $=J_{\mathrm{MS}} P_{\mathrm{SOS}}(0)$ and

$$
\begin{gathered}
J_{\mathrm{MS}}=J_{v} \exp \left[-\left(\left\langle|\Delta \theta|^{2}\right\rangle+\left\langle A^{2}\right\rangle\right) / 2\right], \\
\left\langle|\Delta \theta|^{2}\right\rangle=\frac{1}{2 \beta L^{2}} \sum_{q, i \omega_{n}} \gamma(\vec{q}) G_{0 \theta}\left(\vec{q}, i \omega_{n}\right),
\end{gathered}
$$

$$
\left\langle|A|^{2}\right\rangle=\frac{1}{2 \beta L^{2}} \sum_{q, i \omega_{n}} G_{0 A}\left(\vec{q}, i \omega_{n}\right),
$$

where $\quad G_{0 A}^{-1}\left(\vec{q}, i \omega_{n}\right)=\omega_{n}^{2} / J_{b}+2 K_{b} \gamma(\vec{q})+\left|\omega_{n}\right| / 4 R_{\text {eff }}+J_{\text {eff }}$ and $G_{0 \theta}^{-1}\left(\vec{q}, i \omega_{n}\right)=\omega_{n}^{2} / K_{v}+\gamma(\vec{q})\left(J_{\text {eff }}+\left|\omega_{n}\right| / 4 R_{\text {eff }}\right)$.

The main effect of the gauge fields is to reduce the vortex repulsion $K$. [For weak boson repulsion, $K_{v} \rightarrow K^{*}$ $=\left(K_{v} / 4 \pi^{2}\right)\left(J_{b} / 2 K_{b}\right)\left(h / 4 e^{2} R_{v}\right)$.] Since this is essentially a high-energy cutoff for the physical effects we are considering, it should not affect the critical point for the onset of a finite $J_{\text {eff }}$ for small vortex phase fluctuations. We therefore conclude that this dissipative mechanism will also give rise to a direct superfluid-to-insulator transition in the vortex liquid as $R_{v}$ is increased. Note that the vortex resistance $R_{v}$ is large when the resistance $R_{n}$ of the normal fluid is low. Therefore, we have qualitatively the same zero-temperature behavior here as in the previous model (for direct dissipation from Cooper pair motion) in that the system, in terms of electrical transport by the original Cooper pairs, is superfluid for small $R_{n}$ and insulating for large $R_{n}$. The exact value of the critical resistance is more difficult to extract as it depends in detail on the dependence of $R_{v}$ on $R_{n}$. However, it can be verified that the critical point occurs when $e^{2} R_{n} / h$ is of the order of unity.

At finite temperatures, we again expect crossover behavior. This applies to the insulating side of the (original) SI transition, whereas the crossover behavior of the previous Cooper-pair model affects the superfluid side of the transition. In terms of $R_{n}$ (instead of $R_{v}$ ), this model predicts that, for insulating values of $R_{n}$ (i.e., vortex superfluidity at $T$ $=0$ ), the finite-temperature conductivity for $R_{n}$ may appear metallic or even exhibit signs of (charge) superfluidity above the crossover temperature.

\section{FINITE MAGNETIC FIELD}

Finally, we discuss the effect of a finite magnetic field $B$. This gives rise to a nonzero chemical potential $\mu_{v}$ for vorti- 


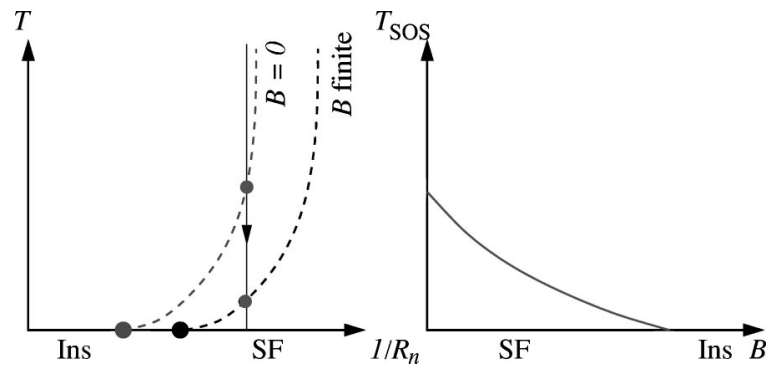

FIG. 3. Finite magnetic field shifts the SOS crossover line (curved-dashed lines). Consider cooling a system near the SI critical point at fixed $R_{n}$ (vertical line in left diagram). The crossover temperature $T_{\text {SOS }}$ is indicated by the circle at the point where the vertical line intersects the SOS crossover line. This decreases with increasing $B$, as sketched in the diagram on the right. Superfluid correlations develop in the system below $T_{\text {Sos }}$. Above $T_{\text {SoS }}$, the system may appear metallic.

ces so that there is a finite density of vortices in the ground state. $\rho_{v}=B / \Phi_{0}$. Again, the movement of the MSOS crossover as a function of vortex density (at fixed $R_{n}$ or $R_{v}$ ) will affect the finite-temperature behavior of the system, and hence the analysis of the experimental data.

Experimentally, we see that the system goes from superconducting to insulating as we increase $B$. Some experiments $^{4,5}$ indicate that there may be a metallic phase between the superconducting and insulating phases. However, there is also evidence 6 at low-applied fields that the metallic behavior only occurs at intermediate temperatures, and that the true zero-temperature phase may be a superconductor after all.

How does this experimental result fit into our description? We speculate that winding-number fluctuations are responsible for this crossover behavior. More specifically, the applied magnetic field increases the winding-number fluctuations in the system in the representation where the bosons are Cooper pairs. (This can be viewed as the bosonic analogue of positive magnetoresistance.) This moves the SOS transition/ crossover line to lower values of $R_{n}$ (to the right in Fig. 1). This is illustrated in Fig. 3. We see that, if the system is near the SI critical point, the crossover temperature $T_{\mathrm{SOS}}$ de- creases rapidly with increasing applied field $B$, as sketched in Fig. 3. This may explain why the crossover from metal to superconductor is only observed experimentally at lowapplied fields ${ }^{6}$.

\section{CONCLUSIONS}

In this paper, we have revisited a model of dissipative Bose systems where conventional theory ${ }^{12}$ predicts a direct superfluid-insulator transition. By treating the phase fluctuations more carefully, we developed a variational approach that can distinguish between superfluid, normal, and insulating phases. We can confirm that a Bose-metal phase does not exist at zero temperature, in agreement with conventional treatment. We are also able to establish the regime of validity for the conventional treatment by studying higher-order effects that couple the small- and large-phase fluctuations.

We have argued that single-parameter scaling might break down because of the influence of large-phase fluctuations (the imaginary-time "winding numbers" of the orderparameter phase). There is a window around the true critical point where strong winding-number fluctuations persist down to exponentially low temperatures. This means that superconductivity may not be observable in this regime at experimentally accessible temperatures. The width of this window of crossover behavior appears to be quite large in our mean-field analysis. We expect that it would be renormalized in a more detailed calculation, and that it would depend on details of the system (such as the degree of disorder).

This window of crossover behavior may be responsible for an apparent metallic phase in some experiments. ${ }^{5}$ The recent observation ${ }^{6}$ of an apparently metallic phase becoming superconducting at very low temperatures appears to support our crossover picture near the superfluid-insulator critical point.

\section{ACKNOWLEDGMENTS}

We would like to thank the British Council for financial support (UK/HK Joint Research Scheme JRS98/40). D.K.K.L. was supported by the Royal Society.
${ }^{1}$ D. B. Haviland, Y. Liu, and A. M. Goldman, Phys. Rev. Lett. 62, 2180 (1989); see also A. M. Goldman and Y. Liu, Physica D 83, 163 (1995).

${ }^{2}$ M. P. A. Fisher, Phys. Rev. Lett. 65, 923 (1990); M. P. A. Fisher, G. Grinstein, and S. M. Girvin, ibid. 64, 587 (1990).

${ }^{3}$ S. Chakravarty, S. Kivelson, G. T. Zimanyi, and B. I. Halperin, Phys. Rev. B 35, 7256 (1987).

${ }^{4}$ H. M. Jaeger, D. B. Haviland, A. M. Goldman, and B. G. Orr, Phys. Rev. B 34, 4920 (1986).

${ }^{5}$ D. Ephron, A. Yazdani, A. Kapitulnik, and B. R. Beasley, Phys. Rev. Lett. 76, 1529 (1996).

${ }^{6}$ N. Mason and A. Kapitulnik, cond-mat/0006138 (unpublished).

${ }^{7}$ M. V. Feigel'man and A. I. Larkin, Chem. Phys. 235, 107 (1998).

${ }^{8}$ D. Das and S. Doniach, Phys. Rev. B 60, 1261 (1999).
${ }^{9}$ J. Bardeen and M. J. Stephen, Phys. Rev. 140, A1197 (1965).

${ }^{10}$ E. Shimshoni, A. Auerbach, and A. Kapitulnik, Phys. Rev. Lett. 80, 3352 (1998).

${ }^{11}$ See N. Mason and A. Kapitulnik, Phys. Rev. Lett. 82, 5341 (1999) for a similar idea.

${ }^{12}$ S. Chakravarty, G. Ingold, S. Kivelson, and A. Luther, Phys. Rev. Lett. 56, 2303 (1986).

${ }^{13}$ See, for example, R. Fazio and G. Schön, Phys. Rev. B 43, 5307 (1991); see also J. E. Mooij, B. J. van Wees, L. J. Geerligs, M. Peters, R. Fazio, and G. Schön, Phys. Rev. Lett. 65, 645 (1990).

${ }^{14}$ V. Ambegaokar, U. Eckern, and G. Schön, Phys. Rev. Lett. 48, 1745 (1982).

${ }^{15}$ A. O. Caldeira and A. J. Leggett, Ann. Phys. (N.Y.) 149, 374 (1983). 
${ }^{16}$ See, for example, G. Schön and A. Z. Zaikin, Phys. Rev. B 40, 5231 (1989).

${ }^{17}$ See, for example, C. Itzykson and J.-M. Drouffe, Statistical Field Theory (Cambridge University Press, Cambridge, 1989), Vol. 1; Y. Saito and H. Müller-Krumbhaar, in Applications of the Monte Carlo Method, edited by K. Binder (Springer-Verlag, Berlin,
1984).

${ }^{18}$ See, for example, S. Sachdev, Quantum Phase Transitions (Cambridge University Press, Cambridge, 1999). If we naively set $N=2$, then the exponential factor is absent.

${ }^{19}$ C. Dasgupta and B. I. Halperin, Phys. Rev. Lett. 47, 1556 (1981); M. P. A. Fisher and D. H. Lee, Phys. Rev. B 39, 2756 (1989). 